\title{
Motor Performance in South African Children with Cystic Fibrosis
}

\section{Abstract}

Aims: This study aimed to investigate motor performance in children with cystic fibrosis (CF) and the relationship with respiratory and anthropometric outcome measures.

Methods: A cross-sectional exploratory study investigated 12 children with CF, mean (SD) age 6.17 (0.67) years, using the Movement Assessment Battery for Children $2^{\text {nd }}$ edition (MABC-2), spirometry, body weight, height, body mass index and age related anthropomorphic z-scores.

Results: MABC-2 total scores indicated 9/12 (75\%) children performed below average $\left(<50^{\text {th }}\right.$ percentile), of which 4/12 children (33.3\%) had motor delay and 2/12 (16.7\%) were at risk for motor delay. The balance subscale showed the lowest scores, with 5/12 (41.7\%) participants performing at or below the $5^{\text {th }}$ percentile and a median (IQR) percentile score of $9.00(5.00-62.50)$.

A significant negative correlation was found between the manual dexterity subscale and both height and height for age $\mathrm{z}$-scores ( $\mathrm{p}=0.017$ and $\mathrm{p}=0.019$ respectively), as well as peak expiratory flow in liter ( $\mathrm{p}=0.027$ ). The balance subscale scores were positively correlated with forced expiratory volume in \%predicted $(\mathrm{p}=0.048)$. No other significant correlations were found.

Conclusion: Children with CF may be at risk for delayed motor development, particularly their balance skills. Poor lung function might affect motor development but further research is recommended.

Keywords: cystic fibrosis, child development, motor skills, South Africa 
Cystic fibrosis (CF) is an autosomal recessive hereditary disease, causing secretory glands throughout the body to produce viscous secretions (Davis, 2006). CF commonly affects the gastrointestinal tract, resulting in nutritional deficits, altered health status and anthropometric characteristics,(Sands, Umławska, \& Zielińska, 2015) and the respiratory system with eventual respiratory failure in advanced disease.(Davis, 2006; Ratjen, 2009)

Exercise and habitual physical activity in children with cystic fibrosis (CF) is known to positively influence lung function capacity, general fitness levels as well as health related quality of life.(Hebestreit et al., 2014; Radtke, Nevitt, Hebestreit, \& Kriemler, 2017) However, children with CF have been reported to have reduced activity levels across the spectrum from high intensity exercise to activities of daily living, compared to their healthy peers.(Wilkes et al., 2009) This is likely to be multifactorial, owing to any or a combination of factors including illness severity and general health status; impaired aerobic and anaerobic fitness levels due to muscle weakness and/or poor respiratory function; poor nutritional status; the perception by many that CF constitutes a barrier to exercise and activity participation; and overprotective parenting styles, which further limit participation.(Arikan, Yatar, Calik-Kutukcu, Aribas, \& Saglam, 2015; Gruet, Troosters, \& Verges, 2017; Nixon, Orenstein, \& Kelsey, 2001; Wilkes et al., 2009)

It is postulated that children with CF may be at risk of delay in motor development owing to participation restriction, however very little is known about motor delay in this population and conflicting evidence can be found in the published literature. A study in infants with CF younger than four years of age, reported that $26.7 \%$ of participants presented with motor delay.(de Almeida Thomazinho, de Miranda Chaves, Pássaro, \& Barbosa Meio, 2011) Another study found that the Munich Fitness Test scores, including items of coordination, were poorer for patients with CF compared to healthy controls.(Arikan et al., 2015) Furthermore, a study by Zengin Akkus et al. (2019) reported delay in fine motor skills, based on the Ages and Stages Questionnaire, when comparing pre-school children with CF to healthy controls.(Zengin Akkus et al., 2019) Conversely, Gruber et al. (2010) reported that pre-school children with CF performed better at motor skills than healthy peers.(Gruber, Orenstein, Paul, Huls, \& Braumann, 2010) Although a significant relation between pulmonary function and physical activity has been found in children with CF,(Schneiderman et al., 2014) no studies have investigated the effect on motor delay in this population. 
This study therefore aimed to describe the motor performance of South African children with $\mathrm{CF}$, aged five to eight years, and the relationship with respiratory and anthropometric outcome measures.

\section{METHODS}

A cross-sectional study was conducted at the CF outpatient clinic of

, a 272-bed tertiary pediatric hospital in

- a low/middle income country. The CF Clinic is a multidisciplinary specialist clinic, which is held weekly, and manages approximately 100 children with CF, from birth to 18 years of age, with the majority living in middle to impoverished socio-economic circumstances.

Institutional ethical approval (HREC 648/2013) and approval from the medical superintendent at the research site was obtained. Written informed consent was obtained from the parent(s) and assent from the children.

\section{Participants}

Children aged five to eight years of age, attending the CF Clinic from January to September 2015 were eligible for inclusion in the study, if they had a confirmed diagnosis of CF, by genotype testing or two positive sweat tests

A convenience sample of 12 children were included in this study.

\section{Measures}

The Movement Assessment Battery for Children second edition (MABC-2), a valid and reliable tool for assessing motor delay in children,(Henderson, Sudgen, \& Barnett, 2007) was used to test the children's motor performance. This tool consists of eight items in three domains: manual dexterity, aiming and catching, and balance; and has normative values for British and Dutch children between the ages of three and 16 years.(Croce, Horvat, \& McCarthy, 2001; Van Waelvelde, De Weerdt, De Cock, \& Smits-Engelsman, 2004) The results are presented as standard scores and percentiles, with color coding red (for percentiles less than five), orange (percentile 9-16), or green (percentile 25 and above). The MABC-2 has been used in research on developmental coordination disorder in South African children, applying the Dutch normative values,(Ferguson, Naidoo, \& Smits-Engelsman, 2015; Jelsma, Ferguson, Smits-Engelsman, \& Geuze, 2015; Smits-Engelsman, Jelsma, Ferguson, \& Geuze, 2015) as there are currently no normative data for the South African population. Therefore, the current study also used the Dutch values. Testing was conducted in a standardized manner 
by the same researcher, at the physiotherapy gym of the outpatient clinic while children were awaiting their doctor's appointment.

The relation between the MABC-2 scores and the following routinely collected outcome measures were investigated: spirometry (forced vital capacity (FVC), forced expiratory volume in one second (FEV 1 ), peak expiratory flow (PEF), forced expiratory flow at 25 and 75\% ( $\left.\mathrm{FEF}_{25-75)}\right)$ conducted by a trained lung function technologist; weight (in kilogram), height (in centimeter), and body mass index (BMI) absolute values measured by the clinic's nurse and values-for-age z-scores as calculated by the WHO AnthroPlus software (available from https://www.who.int/growthref/tools/en/).

\section{Data analysis}

Patient information was de-identified and coded. All data were entered into an Excel spread sheet and analyzed with SPSS (IBM SPSS Statistics for Windows, version 25.0, Armonk, NY, USA). Normality was tested using the Kolmogorov-Smirnov test with Lilliefors significance correction. Numerical data are presented as median and interquartile range (IQR) or mean and standard deviation (SD) as appropriate, and categorical data as n (\%). Comparison with the MABC-2 norms are presented as percentiles of the norm and categorized according to the color code system. Weight, height and BMI for age z-scores were calculated by the use of the WHO AnthroPlus software (available from http://www.who.int/childgrowth/software/en/). Relations between the MABC-2 scores and other factors (spirometry and anthropometric data) were analyzed using one-tailed Pearson or Spearman’s rank correlation coefficients, according to data distribution. Significance level was set at 0.05 .

\section{RESULTS}

Twelve children, median (IQR) age 5.9 (5.7-6.6) years, were included in this study. Participant characteristics are presented in Table 1.

\section{Movement assessment battery for children second edition}

A summary of the percentile scores of the MABC-2 test can be found in Table 2 and Table 3. Overall, 9/12 (75\%) children performed below average $\left(<50^{\text {th }}\right.$ percentile) on the total test score. Balance was the poorest performed subscale, with $5 / 12(41.7 \%)$ performing at the $5^{\text {th }}$ percentile or less (41.7\%) and a median (IQR) percentile of 9.0 (5.0-62.5) (Table 2). 


\section{Movement assessment battery for children-2 and other factors}

The MABC-2 subscale "manual dexterity” was significantly correlated with height, height for age z-scores and PEF (L) (r= -0.62, p=0.017; $\mathrm{r}_{\mathrm{s}}=-0.60, \mathrm{p}=0.019$; and $\mathrm{r}=-0.62, \mathrm{p}=0.027$ respectively). The subscale "balance" on the other hand was significanlty correlated with FVC \%predicted $\left(r_{s}=0.50, p=0.048\right)$. Trends towards significant correlations were found between the total score and age at diagnosis $\left(\mathrm{r}_{\mathrm{s}}=0.49, \mathrm{p}=0.051\right)$. No other significant correlations were found between any of the MABC-2 subscales or total score and either respiratory or anthropmetry factors.

\section{DISCUSSION}

This study found that a large proportion of participants presented with motor delay, mostly notable for the subscale of balance.

These results are in line with findings from de Almeida Thomazinho et al. (2011), although the prevalence of developmental delay in the current study is higher.(de Almeida Thomazinho et al., 2011) This could be due to the older age group of the current study, as delay of gross motor skills might become more prevalent with progression of the disease. However, in a study by Gruber et al. (2008), children aged 6-10 years with CF performed better on all but one task of the modified Munich Fitness Test when compared to children aged 11-15 years and 15-18 years; and all test scores were within lower normal ranges. Arikan et al. (2015) also used the Munich Fitness test, but found, in contrast to Gruber et al (2008), significant differences between children with CF and healthy children on the items "balancing and bouncing a ball”, "hanging score”, and "standing vertical jump”. Although the raw score for "balancing and bouncing a ball” was significantly lower in children with $\mathrm{CF}$, converting scores to age and gender norms made this difference dissapear.(Arikan et al., 2015) This contradicts the current study's findings where the poorest performace was seen on the balance subscale with 9/12 children performing below average compared to the norm values. This may be explained by the different attributes of the balancing exercises used in the Munich Fitness Test (static balance bipodal with external exturbations) and the MABC-2 (static unipodal stance and dynamic walking over a line and jumping/hopping). (Arikan et al., 2015)

Although no research has focussed on balance in children with CF, balance problems might be explained by reduced lung function, as suggested by the results of the current study; as well as decreased peripheral muscle strength as seen in the quadriceps.(Arikan et al., 2015; Gruet et al., 2017) However, no published studies have reported on the strength of other 
stabilising and peripheral muscles, and the impact of muscle strength on gross motor development in children with CF; and such research is stronly recommended. Furthermore, altered body proportions and chest shape (e.g. barrel-shaped chest associated with hyperinflation)(Sands et al., 2015), might change the centre of gravity, thereby impacting on balance. The current study found a positive correlation between \%predicted FVC and balance, indicating that children with higher FVC performed better on the balance subscale. It could therefore be hypothesised that children with better lung capacity might be more active and therefore practice balancing skills in daily life more often. This is supported by previous studies, which have shown that distance walked in 6 minutes is positively associated with FEV 1 and vital capacity.(Gruber, Orenstein, Braumann, \& Huls, 2008; Pereira et al., 2011) The current study also identified a negative correlation between PEF and manual dexterity, which may be due to children with poorer lung function tending to avoid gross motor activities, with a corresponding increase in participation (and thereby improving performance) in more sedentary fine motor activities. The lack of other association between motor development and pulmonary function could be due to the small sample size included in this study. More research is warranted to further investigating the relation between lung functions and motor development.

The correlation between height, height for age, and manual dexterity has not been previously investigated. It may be that taller children were disadvantaged during this aspect of the testing, as the chair and tables used were not adjustable for height, and these children may had to lean forward when performing the tasks. For future research it would be recommended to use a height-adjustable table and chair.

Finally, a trend was found which suggests that children who were diagnosed at an older age performed better on the total MABC-2 test scores. In the context of South Africa, which does not offer newborn screening for CF, it may be that children who are less symptomatic (i.e. healthier) are diagnosed at a later age. Children with fewer symptoms may participate more actively than their symptomatic counterparts, leading to improved motor function. Moreover, the other psychosocial barriers to participation may not be applicable prior to the diagnosis of CF. These suggestions require confirmation larger studies.

This study was limited by the small sample size, the small variation in and relatively unimpaired lung function test results, and the single-centre study design, which also lacks a control group of healthy children as comparators. A further limitation of the current study is 
the use of Dutch norm values rather than comparing to healthy South African children, despite these having been reported in South African studies previously.(Ferguson et al., 2015; Jelsma et al., 2015; Smits-Engelsman et al., 2015) A systematic review on the validity of standardised motor developmental screening tools for babies and toddlers younger then two years of age found that two of the five included studies indicated the need for countryspecific norm values.(Mendonca, Sargent, \& Fetters, 2016) However, none of their included studies were conducted in South Africa, and age range was below that of the current study. In South Africa, the US norm values of the Bayley Scales of Infant Development $3^{\text {rd }}$ edition have been found to be valid for urban Black South African babies.(Rademeyer \& Jacklin, 2013)The fact that three participants obtained scores in the top range of the normative values suggests that the use of the Dutch norms may be valid in the South African population, however this requires further investigation.

\section{CONCLUSIONS}

This preliminary study found a high prevalence of motor delay in children with CF, particularly in their balance skills. This is the first study to report an association between lung function and motor performance. Significant correlations were found between PEF and manual dexterity; and between FVC \%predicted and balance scores. This suggests that lung function may affect motor development and larger trials are recommended to confirm this hypothesis. 


\section{ACKNOWLEDGEMENTS}

We would like to thank all the participants and their parents for participating in this study. We would also like to thank medical staff of the CF clinic at Red Cross War Memorial Children’s Hospital for their support.

\section{CONFLICT OF INTEREST AND SOURCES OF FUNDING}

Dr. Lieselotte Corten was a PhD-student at the Division of Physiotherapy, Department of Health and Rehabilitation Sciences, University of Cape Town, Observatory, Cape Town, South Africa while conducting the research. She received the Margaret Roper Scholarship from the Division of Physiotherapy for her PhD studies at the University of Cape Town. And the Department of Paediatrics and Child Health research award (University of Cape Town) provided funds for this study. The authors have no further conflicts of interest to report. 


\section{References}

Arikan, H., Yatar, I., Calik-Kutukcu, E., Aribas, Z., \& Saglam, M. V.-Y., N; Savci, S; Inal-Ince, D; Ozcelik, U; Kiper, N;. (2015). A comparison of respiratory and peripheral muscle strength, functional exercise capacity, activities of daily living and physical fitness in patients with cystic fibrosis and healthy subjects. Research in Developmental Disabilities, 45-46, 147-156. doi:10.1016/j.ridd.2015.07.020

Croce, R. V., Horvat, M., \& McCarthy, E. (2001). Reliability and concurrent validity of the movement assessment battery for children. Perceptual and Motor Skills, 93(1), 275-280. doi:10.2466/pms.2001.93.1.275

Davis, P. (2006). Cystic fibrosis since 1938. Am J Respir Crit Care Med, 173(5), 475-482. doi:10.1164/rccm.200505-8400E

de Almeida Thomazinho, P., de Miranda Chaves, C., Pássaro, C., \& Barbosa Meio, M. (2011). Motor delay in cystic fibrosis infants: an observational study. Early Human Development, 87 769773. doi:10.1016/j.earlhumdev.2011.05.012

Ferguson, G. D., Naidoo, N., \& Smits-Engelsman, B. C. (2015). Health promotion in a low-income primary school: children with and without DCD benefit, but differently. Physical \& Occupational Therapy In Pediatrics, 35(2), 147-162. doi:10.3109/01942638.2015.1009230

Gruber, W., Orenstein, D., Braumann, K., \& Huls, G. (2008). Health-related fitness and trainability in children with cystic fibrosis. Pediatric Pulmonology, 43, 953-964. doi:10.1002/ppul.20881

Gruber, W., Orenstein, D., Paul, K., Huls, G., \& Braumann, K. (2010). Motor performance is better than normal in preschool children with cystic fibrosis. Pediatric Pulmonology, 45, 527-535. doi:10.1002/ppul.21098

Gruet, M., Troosters, T., \& Verges, S. (2017). Peripheral muscle abnormalities in cystic fibrosis: Etiology, clinical implications and response to therapeutic interventions. Journal of Cystic Fibrosis, 16(5), 538-552. doi:10.1016/j.jcf.2017.02.007

Hebestreit, H., Schmid, K., Kieser, S., Junge, S., Ballmann, M., Roth, K., . . Kriemler, S. (2014). Quality of life is associated with physical activity and fitness in cystic fibrosis. BMC Pulmonary Medicine, 14, 26. doi:10.1186/1471-2466-14-26

Henderson, S., Sudgen, D., \& Barnett, A. (2007). Movement Assessment Battery for Children - Second Edition (examiner's manual).

Jelsma, D., Ferguson, G. D., Smits-Engelsman, B. C., \& Geuze, R. H. (2015). Short-term motor learning of dynamic balance control in children with probable developmental coordination disorder. Res Dev Disabil, 38, 213-222. doi:10.1016/j.ridd.2014.12.027

Mendonca, B., Sargent, B., \& Fetters, L. (2016). Cross-cultural validity of standardized motor development screening and assessment tools: a systematic review. Developmental Medicine \& Child Neurology, 58(12), 1213-1222. doi:10.1111/dmcn.13263

Nixon, P. A., Orenstein, D. M., \& Kelsey, S. F. (2001). Habitual physical activity in children and adolescents with cystic fibrosis. Medicine \& Science in Sports \& Exercice, 33(1), 30-35. doi:10.1097/00005768-200101000-00006.

Pereira, F. M., Ribeiro, M. Â., Ribeiro, A. F., Toro, A. A., Hessel, G., \& Ribeiro, J. D. (2011). Functional performance on the six-minute walk test in patients with cystic fibrosis. Jornal Brasileiro de Pneumologia, 37(6), 735-744. doi:10.1590/\$1806-37132011000600006

Rademeyer, V., \& Jacklin, L. (2013). A study to evaluate the performance of black South African urban infants on the Bayley Scales of Infant Development III. South African Journal of Child Health, 7(2), 54-59. doi:10.7196/sajch.547

Radtke, T., Nevitt, S. J., Hebestreit, H., \& Kriemler, S. (2017). Physical exercise training for cystic fibrosis. Cochrane Database Systematic Reviews, 11, CD002768. doi:10.1002/14651858.CD002768.pub4

Ratjen, F. A. (2009). Cystic fibrosis: pathogenesis and future treatment strategies. Respiratory Care, 54(5), 595-605. 
Sands, D., Umławska, W., \& Zielińska, A. (2015). A cross-sectional study of growth, nutritional status and body proportions in children and adolescents at a medical center specializing in the treatment of cystic fibrosis in Poland. Archives of medical science : AMS, 11(1), 155-163. doi:10.5114/aoms.2015.49207

Schneiderman, J. E., Wilkes, D. L., Atenafu, E. G., Nguyen, T., Wells, G. D., Alarie, N., ... Ratjen, F. (2014). Longitudinal relationship between physical activity and lung health in patients with cystic fibrosis. European Respiratory Journal, 43(3), 817-823. doi:10.1183/09031936.00055513

Smits-Engelsman, B. C., Jelsma, L. D., Ferguson, G. D., \& Geuze, R. H. (2015). Motor learning: an analysis of 100 trials of a ski slalom game in children with and without developmental coordination disorder. PLOS ONE, 10(10), e0140470. doi:10.1371/journal.pone.0140470

Van Waelvelde, H., De Weerdt, W., De Cock, P., \& Smits-Engelsman, B. C. (2004). Aspects of the validity of the Movement Assessment Battery for Children. Human Movement Science, 23(1), 49-60. doi:10.1016/j.humov.2004.04.004

Wilkes, D. L., Schneiderman, J. E., Nguyen, T., Heale, L., Moola, F., Ratjen , F., ... Wells, G. D. (2009). Exercise and physical activity in children with cystic fibrosis. Paediatric Respiratory Reviews, 10(3), 105-109. doi:10.1016/j.prrv.2009.04.001

Zengin Akkus, P., Gharibzadeh Hizal, M., Ilter Bahadur, E., Ozmert, E. N., Eryilmaz Polat, S., Ozdemir, G., .. . Ozcelik, U. (2019). Developmental and behavioral problems in preschool-aged primary ciliary dyskinesia patients. European Journal of Pediatrics, 178(7), 995-1003. doi:10.1007/s00431-019-03382-z 


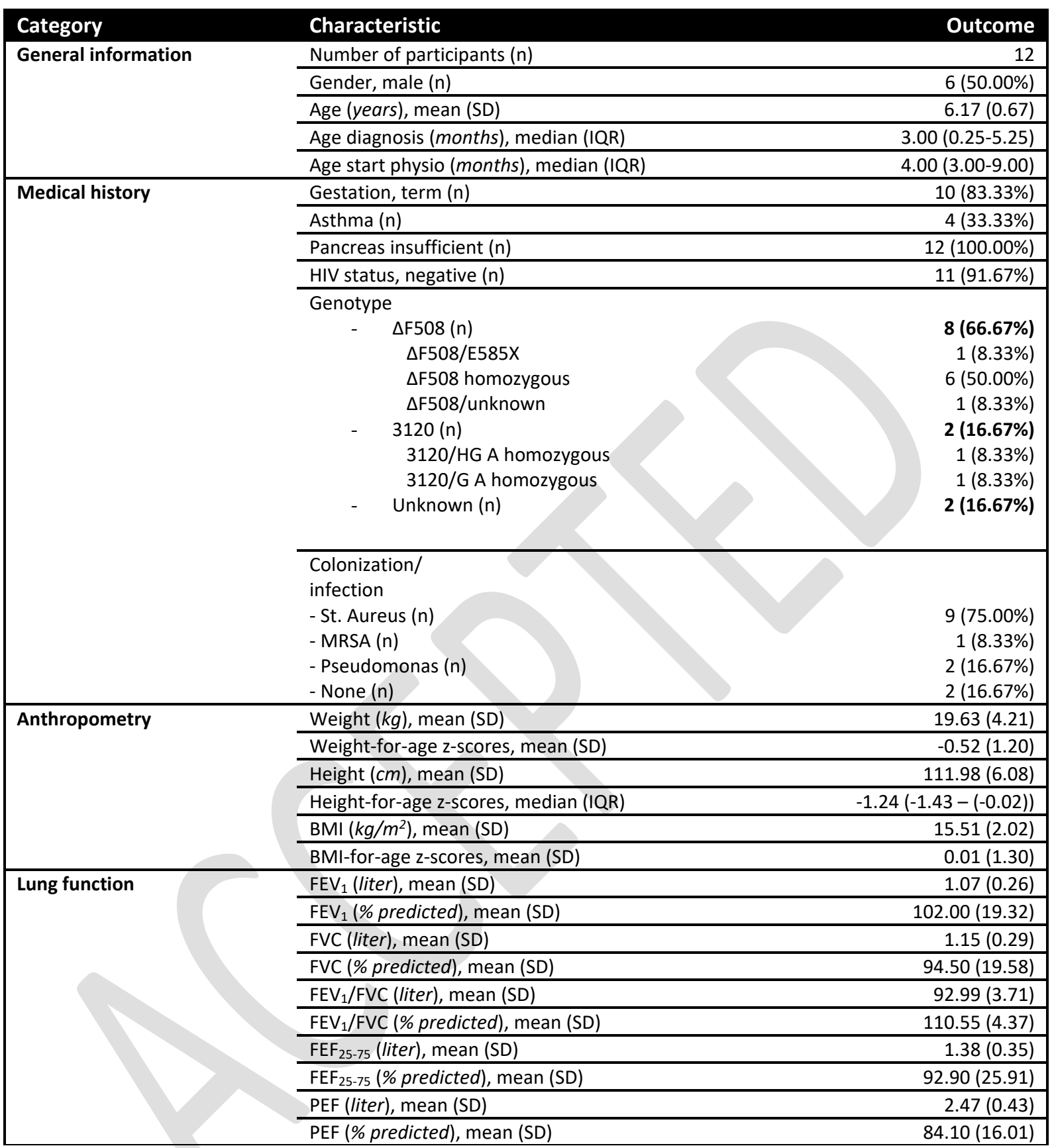

$\mathrm{BMI}=$ body mass index, $\mathrm{FEF}_{25-75}=$ forced expiratory flow at 25 and $75 \%, \mathrm{FEV}_{1}=$ forced expiratory volume in one second $\mathrm{FVC}=$ forced vital capacity, $\mathrm{IQR}=$ interquartile range, $\mathrm{PEF}=$ peak expiratory flow, $\mathrm{SD}=$ standard deviation 
Table 2. Movement assessment battery for children $2^{\text {nd }}$ edition results

\begin{tabular}{|ll|}
\hline MABC-2 category & Outcome \\
\hline Manual dexterity (percentile), mean (SD) & $44.85(40.16)$ \\
\hline Aiming and catching (percentile), mean (SD) & $40.79(30.84)$ \\
\hline Balance (percentile), median (IQR) & $9.00(5.00-62.50)$ \\
\hline Total (percentile), mean (SD) & $34.05(37.54)$ \\
\hline
\end{tabular}


Table 3. Movement assessment battery for children $2^{\text {nd }}$ edition results per participant, presented as percentile

\begin{tabular}{|rrrrr|}
\hline Participant & $\begin{array}{r}\text { Manual } \\
\text { dexterity }\end{array}$ & $\begin{array}{r}\text { Aiming and } \\
\text { catching }\end{array}$ & Balance & Total \\
\hline $\mathbf{1}$ & 91.00 & 84.00 & 75.00 & 95.00 \\
\hline $\mathbf{2}$ & 5.00 & 16.00 & 5.00 & 2.00 \\
\hline $\mathbf{3}$ & 25.00 & 37.00 & 9.00 & 16.00 \\
\hline $\mathbf{4}$ & 99.00 & 75.00 & 91.00 & 98.00 \\
\hline $\mathbf{5}$ & 37.00 & 95.00 & 75.00 & 84.00 \\
\hline $\mathbf{6}$ & 50.00 & 25.00 & 25.00 & 25.00 \\
\hline $\mathbf{7}$ & 0.10 & 25.00 & 0.50 & 0.50 \\
\hline $\mathbf{8}$ & 37.00 & 16.00 & 5.00 & 9.00 \\
\hline $\mathbf{9}$ & 91.00 & 16.00 & 16.00 & 37.00 \\
\hline $\mathbf{1 0}$ & 98.00 & 37.00 & 9.00 & 37.00 \\
\hline $\mathbf{1 1}$ & 0.10 & 0.50 & 0.50 & 0.10 \\
\hline $\mathbf{1 2}$ & 5.00 & 63.00 & 5.00 & 5.00 \\
\hline
\end{tabular}

Dark grey indicates percentiles $\geq 50$, intermediate grey indicates percentiles between 50 and 25 , light grey indicates percentiles between 5 and 25 , and white indicates percentiles $\leq 5$. 